DESY 10-062

\title{
Revisiting fifth forces in the Galileon model
}

\author{
Clare Burrage $^{1}$ and David Seery ${ }^{2}$ \\ 1 Theory Group, Deutsches Elektronen-Synchrotron DESY, D-22603, \\ Hamburg, Germany \\ 2 Department of Physics and Astronomy, University of Sussex, \\ Brighton, BN1 9QH, UK \\ E-mail: clare.burrage@desy.de, D.Seery@sussex.ac.uk
}

\begin{abstract}
A Galileon field is one which obeys a spacetime generalization of the nonrelativistic Galilean invariance. Such a field may possess non-canonical kinetic terms, but ghost-free theories with a well-defined Cauchy problem exist, constructed using a finite number of relevant operators. The interactions of this scalar with matter are hidden by the Vainshtein effect, causing the Galileon to become weakly coupled near heavy sources. We revisit estimates of the fifth force mediated by a Galileon field, and show that the parameters of the model are less constrained by experiment than previously supposed.
\end{abstract}




\section{Introduction}

Many disparate cosmological observations suggest that the universe recently entered an accelerating epoch. The simplest explanation for this acceleration is the inclusion of a cosmological constant $\Lambda \sim\left(10^{-3} \mathrm{eV}\right)^{4}$ in the Einstein equations. Within classical general relativity there is no reason to prefer one value of $\Lambda$ over another. The inclusion of particle physics, however, leads to a natural interpretation of $\Lambda$ as the energy density of the vacuum. Field-theoretic estimates of this energy density diverge as the fourth power of the cutoff, $M$, yielding $\Lambda \sim M^{4}$. A realistic choice almost certainly requires $M \gg \mathrm{TeV}$, which markedly disagrees with the tiny value measured by experiment

The "old" cosmological constant problem is the question: why is the observed value of the cosmological constant so small [1]? But we can just as well ask: why does the vacuum energy gravitate so little [2]? If long wavelength fluctuations gravitate more weakly than other sources, the vacuum energy may be screened or "degravitated," in the same way that long wavelength excitations far beyond the Debye sphere are screened by the effective photon mass in a plasma. By analogy, degravitation occurs if the graviton can acquire a mass, perhaps because it occurs as a resonance [2] or extended object [3], or because it descends from a braneworld scenario such as the DGP model or its generalizations [4, 5]. An implementation of Higgs' mechanism for the graviton has recently been constructed by Chamseddine \& Mukhanov [6].

The four-dimensional effective theory of such models necessarily includes extra scalar fields, arising from new longitudinal polarizations of the graviton. It is these new modes which are the agent of degravitation, requiring them to couple to gravitational sources. Therefore one may have some reservations that these scalars mediate unacceptably large fifth forces. However, it is possible for these fields to avoid experimental constraints using the Vainshtein ("kinetic chameleon") mechanism [7, 8], which exploits their non-canonical kinetic terms. Non-linearities become important in the vicinity of dense objects, and the scalars naturally become weakly coupled to matter. A successful Vainshtein decoupling may require less delicate fine-tuning of parameters.

Nicolis, Rattazzi \& Trincherini [9] discussed the most general four-dimensional ghost-free Lagrangian for a scalar field with these properties. Their construction invoked a gradient shift symmetry for the scalar field, a generalization of non-relativistic Galilei invariance, which permits sizeable non-linearities to occur. Nicolis et al. noticed that a finite set of operators were consistent with this symmetry and simultaneously gave rise to second-order equations of motion. Taking these operators as the relevant terms in an effective field theory, with all other operators considered as irrelevant deformations, one arrives at a perturbatively unitary Lagrangian [10, 11]. Moreover, the relevant operators are protected against renormalization from scalar loops [12, 13].

Nicolis et al. denoted the field described by the resulting Lagrangian as $\pi$, and referred to it as a Galileon. Couplings of the Galileon to gravity were studied by $\ddagger$ If the cutoff for field theory is assumed to be the Planck scale, there is a mismatch between the predicted and observed values of $\Lambda$ by approximately 120 orders of magnitude. 
Deffayet et al. [14, 15], and aspects of its cosmological phenomenology were explored by numerous authors [16, 17, 18, 19, 20]. The gravitational backreaction of the Galileon field was shown to be small in Ref. [21]. An explicit higher dimensional theory, for which the Galileon is the four-dimensional effective theory, was discussed in Ref. [22].

The Galileon Lagrangian contains at least five dimensionful parameters, one for each of the relevant operators \$ These may be chosen freely subject to certain constraints. Of these, the most important is that the acceleration of the Hubble rate should agree with our observations. The remaining parameters must combine in such a way that fifth forces mediated by the Galileon are phenomenologically acceptable. These constraints were discussed by Nicolis et al. [9]. In this paper we revisit this analysis and show that the Vainshtein radius of a massive object - within which the equations of motion of the Galileon become non-linear and the scalar force it mediates is dynamically suppressedcan be made arbitrarily large by varying a single combinations of the parameters. The magnitude of the scalar force within the Vainshtein radius depends on an independent combination of parameters. When a sufficient number of relevant operators are included, these quantities may therefore vary independently.

This freedom leaves the parameters of the Galileon model weakly constrained by gravitational experiments. With this in mind we revisit the strong-coupling limit of the theory and show that, in contrast to the DGP scenario, the distance scale at which non-renormalizable corrections become important may be far below the reach of current experimental probes.

This brief note is organized as follows. In 92 we review the action for a Galileon scalar field. In \$3 the fifth forces mediated by Galileon exchange are calculated, and we demonstrate that the constraints on the parameters of the theory are not as stringent as previously supposed. For this broader parameter space, the scale at which quantum fluctuations become strongly interacting is derived in \$4. We conclude in \$5. In an Appendix we give a simple derivation of the Galileon force law assumed throughout this article. We choose units in which $c=\hbar=1$.

\section{Galileon effective field theories}

It was explained in $\S 1$ that Galileon theories are associated with modifications of gravity in the infrared, owing to the presence of a scalar field, $\pi$, which mixes kinetically with the metric. We are free to choose the precise coupling of $\pi$ to matter, for which various possibilities were discussed by de Rham \& Tolley [22. In Ref. [9] the $\pi$-action was assumed to take the form

$$
S_{\pi}=\int \mathrm{d}^{4} x\left(\mathcal{L}_{\pi}+\pi T^{\mu}{ }_{\mu}\right),
$$

where $T_{\mu \nu}$ is the energy-momentum tensor of matter, which couples to the perturbative graviton $h_{\mu \nu}$ as expected, via $h_{\mu \nu} T^{\mu \nu} / 2$. We allow the self-interactions of $\pi$ to include

$\S$ Other parameters may be associated with irrelevant deformations, which we ignore in the present discussion. 
quite general non-linearities, but treat the coupling between $\pi$ and other fields linearly. In addition, we neglect back-reaction on the metric of any energy density associated with $\pi$. These simplifications are standard in the literature.

The Galilean shift symmetry imposed by Nicolis et al. was

$$
\pi(x) \rightarrow \pi(x)+b_{\mu} x^{\mu}+c,
$$

where $b_{\mu}$ and $c$ are constants. In addition to invariance under this symmetry, as discussed above, the relevant operators in the effective theory for $\pi$ must be chosen to yield secondorder equations of motion. There are five such operators [9]

$$
\begin{aligned}
& \mathcal{L}_{1}=\pi \\
& \mathcal{L}_{2}=-\frac{1}{2} \partial \pi \cdot \partial \pi \\
& \begin{aligned}
\mathcal{L}_{3}=-\frac{1}{2}(\square \pi) \partial \pi \cdot \partial \pi \\
\mathcal{L}_{4}=-\frac{1}{4}\left[(\square \pi)^{2} \partial \pi \cdot \partial \pi-2(\square \pi) \partial \pi \cdot \Pi \cdot \partial \pi\right. \\
\quad-(\Pi \cdot \Pi)(\partial \pi \cdot \partial \pi)+2 \partial \pi \cdot \Pi \cdot \Pi \cdot \partial \pi] \\
\mathcal{L}_{5}=-\frac{1}{5}\left[(\square \pi)^{3} \partial \pi \cdot \partial \pi-3(\square \pi)^{2} \partial \pi \cdot \Pi \cdot \partial \pi-3 \square \pi(\Pi \cdot \Pi)(\partial \pi \cdot \partial \pi)\right. \\
\quad+6(\square \pi) \partial \pi \cdot \Pi \cdot \Pi \cdot \partial \pi+2(\Pi \cdot \Pi \cdot \Pi)(\partial \pi \cdot \partial \pi) \\
\quad+3(\Pi \cdot \Pi) \partial \pi \cdot \Pi \cdot \partial \pi-6 \partial \pi \cdot \Pi \cdot \Pi \cdot \Pi \cdot \partial \pi]
\end{aligned}
\end{aligned}
$$

where '.' indicates contraction of spacetime indices, and $\Pi_{\nu}^{\mu} \equiv \partial^{\mu} \partial_{\nu} \pi$. The relevant part of the $\pi$ effective theory must be constructed out of these operators, making its Lagrangian of the form

$$
\mathcal{L}_{\pi}=\sum_{i=1}^{5} c_{i} \mathcal{L}_{i},
$$

where the $c_{i}$ are arbitrary parameters.

For example, the DGP model [23] is recovered from (8) by the choices $c_{1}=c_{4}=$ $c_{5}=0, c_{2}=24 M_{4}^{2}$ and $c_{3}=16\left(M_{4} / \Lambda\right)^{3}$, where $M_{4}$ is the four dimensional Planck mass, and $\Lambda \sim\left(M_{4} / L_{\mathrm{DGP}}\right)^{1 / 3}$ where $L_{\mathrm{DGP}}$ is the distance scale at which gravity weakens and becomes five dimensional. In a five-dimensional realization of the Galileon model [22], constructed from a four-dimensional DBI probe brane embedded in a five-dimensional spacetime, $c_{2}$ is related to the brane tension, and the higher coefficients $c_{3}, c_{4}$ and

$c_{5}$ receive contributions from the normalization of higher curvature invariants in the five-dimensional brane action. They do not receive perturbative renormalizations from $\pi$ loops [12, 13], although we would generically expect corrections from loops among matter living on the brane. Therefore, in this realization, such corrections may impose naturalness relations among the parameters.

\section{Fifth forces from the Galileon}

If the Galileon model is to give an acceptable phenomenology, it must generate an accelerating universe at late times. Therefore, we expect that $\pi$ has a solution 
corresponding to de Sitter space, even in the absence of sources from other matter fields. Nicolis et al. argued that the necessary solution could be written [9]

$$
\pi_{\mathrm{dS}}(x)=-\frac{1}{4} H^{2} x_{\mu} x^{\mu}
$$

where $H$ is the Hubble parameter of the de Sitter metric. The existence of this solution imposes an algebraic constraint on the coefficients $c_{i}$,

$$
c_{1}-2 c_{2} H^{2}+3 c_{3} H^{4}-3 c_{4} H^{6}+\frac{3}{2} c_{5} H^{8}=0 .
$$

In the presence of matter, the Galileon is perturbed from its background de Sitter solution (9). It is these perturbations which may mediate a fifth force, potentially in conflict with accurate experimental tests of gravity.

\subsection{The Vainshtein radius}

To study solutions of the the Galileon field equations near a massive object, we perturb about the background de Sitter solution

$$
\pi \rightarrow \pi_{\mathrm{dS}}+\pi
$$

in which we have adopted the notation of Nicolis et al. in denoting the Galileon perturbation $\pi$. To avoid ambiguity we use this notation exclusively for the remainder of this article, writing $\pi_{\mathrm{dS}}$ explicitly when we require the background solution and $\pi_{\mathrm{dS}}+\pi$ for the full field.

It follows from (1) and (9) that the equations of motion for $\pi$ are

$$
\sum_{i=2}^{5} d_{i} \mathcal{E}_{i}=-T_{\mu}^{\mu}
$$

where the $d_{i}$ are linear combinations of the $c_{i}$

$$
\left(\begin{array}{l}
d_{2} \\
d_{3} \\
d_{4} \\
d_{5}
\end{array}\right)=\left(\begin{array}{cccc}
1 & -3 H^{2} & \frac{9}{2} H^{4} & -3 H^{6} \\
0 & 1 & -3 H^{2} & 3 H^{4} \\
0 & 0 & 1 & -2 H^{2} \\
0 & 0 & 0 & 1
\end{array}\right)\left(\begin{array}{c}
c_{2} \\
c_{3} \\
c_{4} \\
c_{5}
\end{array}\right)
$$

and the $\mathcal{E}_{i}$ are functions of $\partial_{\mu} \partial_{\nu} \pi$ which coincide with Eqs. (39)-(43) of Ref. [9].

Following Ref. [9], we study the spherically symmetric, static equations of motion for the scalar field near an object of mass $M$ localized at the origin

$$
d_{2}\left(\frac{\pi^{\prime}}{r}\right)+2 d_{3}\left(\frac{\pi^{\prime}}{r}\right)^{2}+2 d_{4}\left(\frac{\pi^{\prime}}{r}\right)^{3}=\frac{M}{4 \pi r^{3}} .
$$

The $\mathcal{L}_{5}$ operator does not contribute to this solution, since it is identically zero when evaluated on a static, radial profile. Therefore $d_{5}$ is unconstrained by this procedure. When $d_{3}=d_{4}=0$ we recover the linear solution

$$
\pi(r)=\pi_{0}-\frac{M}{4 \pi d_{2} r},
$$


for which the ratio of Galileon to gravitational forces is

$$
\frac{F_{\pi}}{F_{G}}=\frac{2 M_{P}^{2}}{d_{2}} .
$$

In this case, modifications of gravity due to the scalar field can be suppressed only by making the Galileon very weakly coupled, requiring $d_{2} \gg M_{P}^{2}$, although in the absence of an ultraviolet completion for general relativity the meaning of masses larger than $M_{P}$ is unclear. If we adopt this strategy, it is hard to justify stability of the resulting scenario against radiative corrections [24.

The situation is very different if at least one of $d_{3}$ or $d_{4}$ is not zero, because nonlinearities in Eq. (14) necessarily become important. We write

$$
\frac{\pi^{\prime}}{r}=\frac{M}{4 \pi r^{3} d_{2}} g(r),
$$

and define two lengthscales, associated with the strength of the $\mathcal{L}_{3}$ and $\mathcal{L}_{4}$ operators and the mass of the object $M$,

$$
\begin{aligned}
R_{1}^{3} & =\frac{d_{3} M}{2 \pi d_{2}^{2}}, \\
R_{2}^{6} & =\frac{M^{2} d_{4}}{8 \pi^{2} d_{2}^{3}} .
\end{aligned}
$$

In terms of these quantities, Eq. (14) can be given a simple expression,

$$
g+\left(\frac{R_{1}}{r}\right)^{3} g^{2}+\left(\frac{R_{2}}{r}\right)^{6} g^{3}=1 .
$$

The $\pi$-profile sources a $1 / r^{2}$ force law whenever $g=1$. The strength of this force is set by $d_{2}$, which has dimensions of $\left[\mathrm{GeV}^{2}\right]$, giving an effective 'Galileon Newton's constant' $G_{\pi} \sim 1 / d_{2}$. This $1 / r^{2}$ force will be stronger than gravity - and therefore in potential conflict with observation - if $d_{2} \lesssim M_{\mathrm{P}}^{2}$. However, the force can be made substantially weaker if $g$ becomes much smaller than unity.

When can we expect significant suppression? Eq. (20) shows clearly that, whenever $R_{1}$ or $R_{2}$ are non-zero, the solution $g(r)$ must approach zero sufficiently rapidly as $r \rightarrow 0$. Therefore, Eq. (20) automatically suppresses the $\pi$-force within a radius $r<r_{\star}$, where the strong-coupling or 'Vainshtein' radius $r_{\star}$ is controlled by the onset of non-linear behaviour in Eq. (20). To obtain an estimate one can determine the radius $r_{n}$ at which $g$ has decreased to $\sim 1 / n$, where $n$ is a small natural number,

$$
r_{n}^{3}=\frac{R_{1}^{3}+\sqrt{R_{1}^{6}+4(n-1) R_{2}^{6}}}{2 n(n-1)} .
$$

If any hierarchy exists between $R_{1}$ and $R_{2}$, then $r_{n}$ is approximately given by the larger of the two. On the other hand, if $R_{1} \sim R_{2}$ then $r_{n} \sim R_{1} \sim R_{2}$. In either case we can make a good estimate of the Vainshtein radius by setting $r_{\star} \sim \max \left\{R_{1}, R_{2}\right\}$. This remarkable, automatic suppression of the $\pi$-force for $r<r_{\star}$ is the basis of the Vainshtein mechanism, allowing fifth forces to be dynamically suppressed without fine-tuning parameters.

It is possible to set $R_{1}$ and $R_{2}$ to arbitrary values by varying the Lagrangian coefficients $c_{1}, c_{2}$ and $c_{3}$, which allows the Vainshtein radius to be made arbitrarily 
large or small. This is more general than Vainshtein's original scenario [7]. It is also more general than the first non-trivial Galileon scenario, which was realized within the DGP model [23]. In this case the Vainshtein radius for an object of mass $M$ depends only on the Planck scale, $M_{\mathrm{P}}$, and the scale of the de Sitter cosmological constant,

$$
r_{*} \sim\left(\frac{M}{4 \pi} \frac{1}{M_{\mathrm{P}}^{2} H_{0}^{2}}\right)^{1 / 3} .
$$

This happens because the DGP model fixes the Lagrangian parameters to be combinations of the Planck and Hubble scales up to $\mathrm{O}(1)$ constants.

\subsection{Suppression within the Vainshtein radius}

We have seen that non-linearities inherent in the Galileon model dynamically suppress $\pi$-exchange forces within the Vainshtein radius of a massive object; the suppression factor $g(r)$ in Eq. (17) must tend to zero as the origin is approached. In this section we argue that if the 'bare' coupling constant of the far-field $1 / r^{2}$ force is too large, so that $d_{2} \ll M_{P}^{2}$, dynamical suppression may not be enough to hide the Galileon from experimental probes of gravity, which are conducted at a finite distance from the origin. It is necessary to apply a further restriction to the Lagrangian parameters, controlling the degree of suppression when $r \ll r_{\star}$.

We first compute the Galileon force within the Vainshtein region. In Ref. [9] it was argued that stable solutions to the equations of motion exist only if $d_{2}>0, d_{4} \geqslant 0$, $d_{3} \geqslant \sqrt{(3 / 2) d_{2} d_{4}}$ and $d_{5}<0$. This entails the relation $R_{1} \geqslant 3^{1 / 6} R_{2}>R_{2}$. Also, it is useful to introduce a quantity $\alpha$ which measures the hierarchy between $\mathcal{L}_{3}$ and $\mathcal{L}_{4}$ in terms of their associated radii $R_{1}$ and $R_{2}$,

$$
\alpha \equiv\left(\frac{R_{2}}{R_{1}}\right)^{3}<1 .
$$

If both $R_{1}$ and $R_{2}$ are non-zero, there are two spatial regions in which the solution can be approximated easily:

- Region A: $\alpha R_{2} \ll r \ll R_{1}$.

This implies $g \sim\left(r / R_{1}\right)^{3 / 2}$, meaning that the ratio of the scalar $\left(F_{\pi}\right)$ to gravitational $\left(F_{N}\right)$ force is

$$
\frac{F_{\pi}}{F_{N}}=\frac{M_{P}^{2}}{4 \pi d_{2}}\left(\frac{r}{R_{1}}\right)^{3 / 2}=\frac{M_{P}^{2}}{2}\left(\frac{1}{2 \pi d_{3} M}\right)^{1 / 2} r^{3 / 2} .
$$

- Region B: $0<r \ll \alpha R_{2}$.

In this region $g \sim\left(r / R_{2}\right)^{2}$, meaning that the ratio of the scalar to gravitational force is

$$
\frac{F_{\pi}}{F_{N}}=\frac{M_{P}^{2}}{4 \pi d_{2}}\left(\frac{r}{R_{2}}\right)^{2}=\frac{M_{P}^{2}}{2}\left(\frac{1}{\pi d_{4} M^{2}}\right)^{1 / 3} r^{2}
$$

Clearly Region B only exists when $d_{4} \neq 0$. In principle, Region B could extend from zero to $r \sim r_{\star}$ if $d_{3}$ is absent. However, this arrangement is excluded by the stability argument discussed above which requires $R_{1}>R_{2}$. 
Eqs. (24)-(25) show clearly that for $r<r_{\star}$, the Galileon force is independent of $d_{2}$. This effect was noticed very recently by Gannouji \& Sani [20]. The interpretation is simple: the scale $d_{2}$ controls the strength of the Galileon force in the linear regime, where $\pi$-exchange obeys a $1 / r^{2}$ force law. Therefore it is clear that $d_{2} \gtrsim M_{P}^{2}$ is sufficient to ensure Galileon forces are suppressed within the Vainshtein radius, because whenever $r$ falls appreciably below $r_{\star}$ we will find that $F_{\pi} / F_{N}$ becomes negligible. This choice was made in Ref. [9].

However, this is not necessary. The linear region, where a $1 / r^{2}$ force law prevails, may never probed be directly by experimental tests of gravity. Instead, experiments typically study the motion of a test mass in the gravitational field of a much more massive source. What conditions must we impose? First, if the Galileon is to hide dynamically from experiments seeking modifications of gravity, then the entire experiment must lie within its own Vainshtein radius. Second, Eqs. (24)-(25) for the Galileon force show that, provided we know the separation of source and test masses, and the source mass $M$, then gravitational tests constrain either $d_{3}$ or $d_{4}$. For consistency we must ensure that the Vainshtein radius of the source mass is sufficiently large to contain the experimental apparatus - requiring a tuning of the independent combinations of Lagrangian parameters in $R_{1}$ and $R_{2}$.

These constraints do not ensure that the Galileon force is weaker than gravity everywhere within the Vainshtein radius. Indeed, for $r \sim r_{\star}$ we find $F_{\pi} / F_{N} \sim M_{\mathrm{P}}^{2} / d_{2}$ which may be large. However, the freedom to make the Vainshtein radius of any object arbitrarily large means it is never necessary to impose the much more stringent condition that $F_{\pi} / F_{N} \ll 1$ for all $r<r_{\star}$. We can therefore extend the parameter range which was allowed in Ref. [9] to include the region where $d_{2}<M_{\mathrm{P}}^{2}$, without damage to a successful Vainshtein decoupling. For example, in the de Rham-Tolley construction discussed in $\$ 2$ one has $c_{2} \sim \lambda$, where $\lambda$ is the tension of the 3-brane. In this construction, we conclude that even in backgrounds where $c_{2} \sim d_{2}$, the tension may be adjusted as desired, without being constrained to the Planck scale.

\subsection{Gravitationally bound objects}

We leave detailed calculation of the constraints imposed by laboratory and astronomical tests for future work. However, we can obtain an estimate of the freedom allowed in $d_{2}, d_{3}$ and $d_{4}$ by imposing a simple and generic requirement: gravitationally bound objects should not feel significant deviations from general relativity. This is necessary because the validity of post-Newtonian gravity has been established for a wide variety of gravitationally bound systems.

We denote the Ricci curvature $\mathcal{R}$ and approximate it near a massive object by $\mathcal{R} \sim R_{\mathrm{S}} / r^{3}$, where $R_{\mathrm{S}}$ is the Schwarzschild radius of the object. A system is gravitationally bound if its gravitational attraction is stronger than the Hubble flow, which requires $\mathcal{R} \gtrsim H_{0}^{2}$. We can reformulate this constraint by asking that the system 
is entirely contained within the radius

$$
r \lesssim R_{\text {bound }} \equiv\left(\frac{R_{\mathrm{S}}}{H_{0}^{2}}\right)^{1 / 3}
$$

According to the argument outlined in $\lceil 3.2$, if the Galileon is to evade experimental probes of gravity dynamically then we must require that $R_{\text {bound }}$ is smaller than the radius at which the gravitational and Galileon forces are equal in magnitude

$$
R_{\text {equality }}=8 \pi \frac{d_{3} M}{M_{P}^{4}} .
$$

The requirement $R_{\text {bound }} \lesssim R_{\text {equality }}$ therefore imposes the constraint

$$
d_{3} \gtrsim 10^{118} .
$$

This can be written as a constraint on the Lagrangian parameters by making use of Eq. (13). For consistency, to ensure that the Vainshtein radius is at least as large as $R_{\text {equality }}$, we must also require $d_{2} \lesssim M_{P}^{2}$.

In Ref. [9] it was argued that for gravitationally bound objects to be within their own Vainshtein radius it was necessary to have $d_{2} \sim M_{P}^{2}$. Here we have demonstrated that, because in the Galileon model we have the freedom to vary the Vainshtein radius of an object, this is too restrictive a constraint.

\section{The strong interaction scale}

In the DGP model the Lagrangian parameters $c_{i}$ are constrained by the five-dimensional origin of the theory, and are given by combinations of the four-dimensional Planck and Hubble scales. This naïvely determines the scale at which non-renormalizable operators provide important corrections. For example, when calculating the Newtonian potential, this reasoning suggests that non-renormalizable operators become important near energies $\gtrsim 1 / \ell[25$, where $\ell \sim 1000 \mathrm{~km}$. In principle one should not trust computations of the classical gravitational potential at distances $\lesssim \ell$. However, it has long been known that an effect similar to the Vainshtein mechanism suppresses these unwanted corrections, making the theory predictive at least to centimetre scales. In this section we will demonstrate that our freedom to set the Lagrangian parameters of the Galileon effective theory effectively means that corrections can be made to enter at almost arbitrarily high scales.

For clarity we denote fluctuations around the classical solution by $\phi$, so that the full field can be written $\pi_{\mathrm{dS}}+\phi$. Making a field redefinition $\hat{\phi}=d_{2}^{1 / 2} \phi$, the action for $\hat{\phi}$ can be written schematically

$$
\mathcal{L}_{\hat{\pi}}=-(\partial \hat{\phi} \cdot \partial \hat{\phi})\left(\frac{1}{2}+\frac{1}{2} \frac{d_{3}}{d_{2}^{3 / 2}}[P]+\frac{1}{4} \frac{d_{4}}{d_{2}^{2}}[P]^{2}+\frac{1}{5} \frac{d_{5}}{d_{2}^{5 / 2}}[P]^{3}\right),
$$

where all indices have been suppressed and $[P]$ indicates a series of terms of the form $\partial \partial \hat{\phi}$, with the indices variously contracted. On inspection of this Lagrangian we might 
expect strong interactions to enter near any or all of the energies $\sim d_{2}^{1 / 2} / d_{3}^{1 / 3}, \sim d_{2}^{1 / 3} / d_{4}^{1 / 6}$ or $\sim d_{2}^{5 / 18} / d_{5}^{1 / 9}$.

In the Galileon scenario these estimates can be rather misleading. It was explained in $\$ 3.2$ that no gravitational experiment is sensitive to fluctuations around a de Sitter background; instead, such experiments measure fluctuations in the neighbourhood of massive objects. Within the Vainshtein radius, renormalizations coming from the background significantly alter the strong interaction scale. For illustration, consider the case where $c_{4}=c_{5}=d_{4}=d_{5}=0$. The Lagrangian for quantum fluctuations $\phi$ about the solution for the $\pi$-field around a massive body is

$$
\mathcal{L}_{\phi}=Z_{\mu \nu} \partial^{\mu} \phi \partial^{\nu} \phi-\frac{d_{3}}{2}(\square \phi) \partial \phi \cdot \partial \phi
$$

where

$$
Z_{\mu \nu}=-\frac{d_{2}}{2} \eta_{\mu \nu}-d_{3} \square \pi \eta_{\mu \nu}+d_{3} \partial_{\mu} \partial_{\nu} \pi
$$

Deep inside the Vainshtein radius of a massive object, where $r \ll R_{1}$, all non-zero entries of $Z_{\mu \nu}$ become large and of the same order. We will discuss $Z_{\mu \nu}$ in more detail below. In the present case we are assuming $d_{4}=0$, and there is no Region B. Therefore, within the Vainshtein radius, the $r$-dependence $\pi$ is determined by the $\mathcal{L}_{3}$ operator, and the non-zero elements of $Z_{\mu \nu}$ are of an approximately common magnitude $Z$,

$$
Z \sim-\frac{d_{2}}{2}\left[1-\frac{1}{2}\left(\frac{R_{1}}{r}\right)^{3 / 2}\right] .
$$

When $Z$ is large, this raises the effective quantum cutoff to $\tilde{\Lambda}(x) \sim Z^{1 / 2} / d_{3}^{1 / 3}$.

The improvement is significant. In computations of the Newtonian potential at the surface of the Earth, this raises the cutoff in the DGP model to $\tilde{\Lambda}(x) \sim 1 /(1 \mathrm{~cm})[25]$. Unfortunately, this is two orders of magnitude lower than the scales probed in current gravitational experiments, which can reach $\sim 10^{-2} \mathrm{~cm}$. It is not yet clear whether this is in conflict with observation. In the Galileon model with $c_{4}=c_{5}=0$, the bound given in Eq. (28) implies that we also find

$$
\tilde{\Lambda} \lesssim \frac{1}{1 \mathrm{~cm}}
$$

Therefore, in this model, our freedom to change the Lagrangian parameters does not significantly improve our control over non-renormalizable operators.

The situation is different in the full Galileon model with all the $c_{i}$ non-zero. The possibility of a non-trivial Region $\mathrm{B}$ emerges and we have more freedom. Within the Vainshtein radius, the behaviour of $\pi$ as a function of $r$ depends whether $\mathcal{L}_{3}$ or $\mathcal{L}_{4}$ dominates its dynamics. Therefore, the strong coupling scale will vary between Region A and Region B. It follows from the expressions given in Ref. [9] that the quadratic Lagrangian for small perturbations about $\pi$ satisfies

$$
S_{\phi}=\frac{1}{2} \int d^{4} x\left[Z_{t}(r)\left(\partial_{t} \phi\right)^{2}-Z_{r}(r)\left(\partial_{r} \phi\right)^{2}-Z_{\Omega}(r)\left(\partial_{\Omega} \phi\right)^{2}\right]
$$


where $\partial_{\Omega}$ denotes derivatives with respect to angular variables. The $Z$ coefficients take different forms in the two Regions A and B but, as with the simple example discussed above, within the Vainshtein radius they are always much larger than the naïve scale $d_{2}$.

- Region A: The $Z$ coefficients scale as

$$
\begin{aligned}
& Z_{r} \sim 2 d_{2}\left(\frac{R_{1}}{r}\right)^{3 / 2} \\
& Z_{t} \sim \frac{3 d_{2}}{2}\left(\frac{R_{1}}{r}\right)^{3 / 2}-\frac{3 d_{5} d_{2}^{3}}{2 d_{3}^{3}}\left(\frac{R_{1}}{r}\right)^{9 / 2} \\
& Z_{\Omega} \sim \frac{d_{2}}{2}\left(\frac{R_{1}}{r}\right)^{3 / 2}
\end{aligned}
$$

If the contribution proportional to $d_{5}$ is negligible, then all of the $Z_{i}$ have the same behaviour. This is analogous to the simple example with $c_{4}=c_{5}=0$, discussed above. The lower bound on $d_{3}$ given by Eq. (28) means that at fixed radius $r$, the $Z_{i}$ are bounded above and cannot be made arbitrarily large. Therefore, at that point the strong coupling scale cannot be pushed to arbitrarily small distances.

- Region B: The $Z$ coefficients scale as

$$
\begin{aligned}
Z_{r} & \sim 3 d_{2}\left(\frac{R_{2}}{r}\right)^{2} \\
Z_{t} & \sim 3 d_{2}\left(\frac{R_{2}}{r}\right)^{2}-\frac{d_{5} d_{2}^{3 / 2}}{\sqrt{2} d_{4}^{3 / 2}}\left(\frac{R_{2}}{r}\right), \\
Z_{\Omega} & \sim-d_{2}+\frac{2 d_{3}^{2}}{3 d_{4}}
\end{aligned}
$$

In this region the $Z_{i}$ scale differently with $r$. We see that by varying the $d_{i}$ it is possible to make all of these coefficients arbitrarily large.

In a general Galileon theory, where all of the $c_{i}$ are non-zero, not only $\mathcal{L}_{2}$ will be renormalized, but also $\mathcal{L}_{3}$ and $\mathcal{L}_{4}$. Therefore it is unclear which term in Eq. (29) gives rise to the leading order strong-coupling corrections, because we have no understanding of the natural scales for each $c_{i}$. For example, if the leading quantum corrections come from the $\mathcal{L}_{5}$ operator, then the strong coupling scale is $\sim Z(r)^{5 / 18} / d_{5}^{1 / 9}$. We have seen above that the form of $Z(r)$ depends whether the point of evaluation lies in Region A or Region $\mathrm{B}$, but it is clear that in either regime we are free to vary the Lagrangian parameters so that the strong coupling scale lies beyond the reach of current experiments.

\section{Conclusions}

The Galileon is a scalar field coupled to the matter content of the Standard Model. Its effects can be hidden from searches for modifications of gravity, without gross finetuning of parameters, because its non-linear self-interactions cause the field to become 
weakly coupled near heavy objects. The form of these self interactions is protected by a derivative shift symmetry.

Our discussion has been framed in the context of an effective field theory, where the Galileon's behaviour is controlled by a small number of relevant couplings. In some previous discussions, compatibility with a higher-dimensional completion has fixed these couplings and therefore the Vainshtein radius. In Ref. 9] the couplings were allowed to vary freely, but the far-field interaction strength, controlled by the coupling $d_{2}$, was set to gravitational strength. In this article we have shown that the Vainshtein radius associated with a massive object can be made arbitrarily large or small by varying a linear combination of the Lagrangian parameters. Experiments probing the force on a test mass interacting with a heavy source at fixed radius can be made insensitive to Galileon exchange forces by varying an independent linear combination of Lagrangian parameters. In principle this allows the far-field interaction strength to be made much stronger. Whether such an arrangement can be technically natural is presently unknown.

Calculation of the precise constraints imposed by experimental tests of gravity is left for future work. However, it seems reasonable that observations do not force us to put all the Lagrangian parameters at the Planck scale, as had been supposed. Instead, they may vary widely over many orders of magnitude. This is particularly interesting in light of the recently proposed five-dimensional origin of the Galileon model [22], where the Galileon parameters derive from combinations of the parameters of the five-dimensional theory. The freedom to vary the Galileon parameters also means that the quantum strong coupling scale can be made arbitrarily high, and placed out of reach of current experimental probes.

\section{Acknowledgments}

We would like to thank Claudia de Rham and Andrew Tolley for very helpful discussions. $\mathrm{CB}$ is supported by the German Science Foundation (DFG) under the Collaborative Research Centre (SFB) 676, and would like to thank the University of Sussex for their hospitality while this work was completed. DS is supported by STFC.

\section{Appendix A. Force on a test particle}

To describe the effects of the Galilean field on a test particle we determine its geodesic equation, including the influence of mixing between $\pi$ and the metric. In the Galileon theory we treat the gravitational field, and $\pi$ 's contribution to it, at linear order, and measure length along the worldline of a point particle using the metric $g_{a b}=(1+2 \pi) \eta_{a b}$, [9]. The action of a point particle of mass $m$ is

$$
S=-m \int \mathrm{d} \tau \sqrt{1+2 \pi}\left(-\dot{X}^{a} \dot{X}_{a}\right)^{1 / 2}
$$

where the $X^{a}$ describe an embedding of the particle's worldline into spacetime, parametrized by $\tau$. Variation of this action leads us to the Galileon analogue of the 
geodesic equation

$$
\frac{d u^{b}}{d \tau}=-\nabla_{a} \pi\left[\delta_{b}^{a}\left(-\dot{X}^{c} \dot{X}_{c}\right)^{1 / 2}+u^{a} u^{b}\right]
$$

where $u^{a}=\dot{X}^{a} / \sqrt{-\dot{X}^{b} \dot{X}_{b}}$. In 93 we considered only static, spherically symmetric solutions for $\pi$. For such solutions we see from (A.2) that the force per unit mass on a point particle due to the Galileon field is

$$
F_{\pi}=\frac{\mathrm{d} \pi(r)}{\mathrm{d} r}
$$

\section{References}

[1] S. Weinberg, The cosmological constant problems, astro-ph/0005265.

[2] G. Dvali, S. Hofmann, and J. Khoury, Degravitation of the cosmological constant and graviton width, Phys. Rev. D76 (2007) 084006, hep-th/0703027.

[3] R. Sundrum, Fat gravitons, the cosmological constant and sub- millimeter tests, Phys. Rev. D69 (2004) 044014, hep-th/0306106.

[4] C. de Rham et. al., Cascading gravity: Extending the Dvali-Gabadadze-Porrati model to higher dimension, Phys. Rev. Lett. 100 (2008) 251603, arXiv:0711.2072.

[5] C. de Rham, S. Hofmann, J. Khoury, and A. J. Tolley, Cascading Gravity and Degravitation, JCAP 0802 (2008) 011, arXiv:0712.2821.

[6] A. H. Chamseddine and V. Mukhanov, Higgs for Graviton: Simple and Elegant Solution, arXiv:1002.3877

[7] A. I. Vainshtein, To the problem of nonvanishing gravitation mass, Phys. Lett. B39 (1972) 393-394.

[8] C. Deffayet, G. R. Dvali, G. Gabadadze, and A. I. Vainshtein, Nonperturbative continuity in graviton mass versus perturbative discontinuity, Phys. Rev. D65 (2002) 044026, hep-th/0106001.

[9] A. Nicolis, R. Rattazzi, and E. Trincherini, The galileon as a local modification of gravity, Phys. Rev. D79 (2009) 064036, arXiv:0811.2197.

[10] J. F. Donoghue, General relativity as an effective field theory: The leading quantum corrections, Phys. Rev. D50 (1994) 3874-3888, gr-qc/9405057.

[11] J. F. Donoghue, Leading quantum correction to the Newtonian potential, Phys. Rev. Lett. 72 (1994) 2996-2999, gr-qc/9310024.

[12] M. A. Luty, M. Porrati, and R. Rattazzi, Strong interactions and stability in the DGP model, JHEP 09 (2003) 029, hep-th/0303116.

[13] M. Porrati and J. W. Rombouts, Strong coupling vs. 4-D locality in induced gravity, Phys. Rev. D69 (2004) 122003, hep-th/0401211.

[14] C. Deffayet, G. Esposito-Farese, and A. Vikman, Covariant Galileon, Phys. Rev. D79 (2009) 084003, arXiv:0901.1314.

[15] C. Deffayet, S. Deser, and G. Esposito-Farese, Generalized Galileons: All scalar models whose curved background extensions maintain second-order field equations and stress-tensors, Phys. Rev. D80 (2009) 064015, arXiv:0906.1967.

[16] N. Chow and J. Khoury, Galileon Cosmology, Phys. Rev. D80 (2009) 024037, arXiv:0905.1325.

[17] F. P. Silva and K. Koyama, Self-Accelerating Universe in Galileon Cosmology, Phys. Rev. D80 (2009) 121301, arXiv:0909.4538.

[18] T. Kobayashi, Cosmic expansion and growth histories in Galileon scalar- tensor models of dark energy, arXiv:1003.3281. 
[19] T. Kobayashi, H. Tashiro, and D. Suzuki, Evolution of linear cosmological perturbations and its observational implications in Galileon-type modified gravity, Phys. Rev. D81 (2010) 063513, arXiv:0912.4641.

[20] R. Gannouji and M. Sami, Galileon gravity and its relevance to late time cosmic acceleration, arXiv: 1004.2808

[21] K. Van Acoleyen, On the cosmological backreaction for large distance modifications of gravity, arXiv:0907.4116

[22] C. de Rham and A. J. Tolley, DBI and the Galileon reunited, arXiv:1003.5917.

[23] G. R. Dvali, G. Gabadadze, and M. Porrati, 4D gravity on a brane in 5D Minkowski space, Phys. Lett. B485 (2000) 208-214, hep-th/0005016.

[24] S. M. Carroll, Quintessence and the rest of the world, Phys. Rev. Lett. 81 (1998) 3067-3070, astro-ph/9806099.

[25] A. Nicolis and R. Rattazzi, Classical and quantum consistency of the DGP model, JHEP 06 (2004) 059, hep-th/0404159. 